\title{
Identification and function analysis of contrary genes in Dupuytren's contracture
}

\author{
XIANGLU JI, FENG TIAN and LIJIE TIAN \\ Department of Hand and Foot Surgery, Shengjing Hospital, China Medical University, \\ Shenyang, Liaoning 110003, P.R. China
}

Received May 16, 2014; Accepted January 29, 2015

DOI: $10.3892 / \mathrm{mmr} .2015 .3458$

\begin{abstract}
The present study aimed to analyze the expression of genes involved in Dupuytren's contracture (DC), using bioinformatic methods. The profile of GSE21221 was downloaded from the gene expression ominibus, which included six samples, derived from fibroblasts and six healthy control samples, derived from carpal-tunnel fibroblasts. A Distributed Intrusion Detection System was used in order to identify differentially expressed genes. The term contrary genes is proposed. Contrary genes were the genes that exhibited opposite expression pattterns in the positive and negative groups, and likely exhibited opposite functions. These were identified using Coexpress software. Gene ontology (GO) function analysis was conducted for the contrary genes. A network of GO terms was constructed using the reduce and visualize gene ontology database. Significantly expressed genes (801) and contrary genes (98) were screened. A significant association was observed between Chitinase-3-like protein 1 and ten genes in the positive gene set. Positive regulation of transcription and the activation of nuclear factor $-\kappa \mathrm{B}(\mathrm{NF}-\kappa \mathrm{B})$-inducing kinase activity exhibited the highest degree values in the network of GO terms. In the present study, the expression of genes involved in the development of DC was analyzed, and the concept of contrary genes proposed. The genes identified in the present study are involved in the positive regulation of transcription and activation of $\mathrm{NF}-\kappa \mathrm{B}$-inducing kinase activity. The contrary genes and GO terms identified in the present study may potentially be used for DC diagnosis and treatment.
\end{abstract}

\section{Introduction}

Dupuytren's contracture (DC) is a progressive tissue disorder that affects the palmar fascia, and results in digital flexion

Correspondence to: Dr Xianglu Ji, Department of Hand and Foot Surgery, Shengjing Hospital, China Medical University, 36 Sanhao Street, Shenyang, Liaoning 110003, P.R. China E-mail: xiangluji001@163.com

Key words: Dupuytren's contracture, contrary genes, gene ontology term, network contracture (1-3). DC incidence ranges from 3-40\% worldwide and primarily affects patients $>50$ years (4). Therefore, it is necessary to investigate novel diagnostic and therapeutic approaches for patients with DC.

A number of treatments for DC have been evaluated, such as radiotherapy, topical vitamin $\mathrm{A}$ and $\mathrm{E}$ application, and dimethyl sulfoxide injection. However, these approaches have proven unsuitable or ineffective for the clinical treatment of DC (5). More recently, research has focused on potential treatments targeting the molecular processes underlying DC $(6,7)$. Fibrogenic cytokines, which may be capable of inducing the growth of fibroblasts, are involved in the molecular mechanisms underlying DC (8). Satish et al (9) demonstrated that the expression of type XV collagen $\alpha 1$ chain, proteoglycan 4 and fibulin-1, which are components of the extracellular matrix, is downregulated in DC fibroblasts compared with controls. The following miRNAs were found to be associated with the $\beta$-catenin pathway in DC tissue samples: Zinc finger of the cerebellum 1, wingless-type mouse mammary tumor virus integration site $5 \mathrm{~A}$ and transforming growth factor $\beta 1$, (10). Musculoaponeurotic fibrosarcoma oncogene homolog B has been reported to be expressed in DC samples and not in healthy samples (11). Furthermore, a number of matrix metalloproteinases (MMPs), including MMP 13, MMP 16 and MMP 19 are expressed in DC samples but not in healthy samples (12). However, the pathogenesis of DC remains poorly understood. It is therefore necessary to investigate the pathology of DC in order to develop diagnostic and therapeutic approaches to DC.

In the present study, statistical analysis was performed, in order to develop gene expression profiles for samples from patients with DC and from healthy controls. The significantly differentially expressed genes (DEGs) between DC patients and healthy controls were measured. Hierarchical clustering analysis was performed for the DEGs. Antagonistic contrary genes, which exhibited opposite expression patterns in the positive and negative groups, and likely exhibited opposite functions, were then identified. A gene ontology (GO) function analysis was conducted and a network of GO terms was constructed.

\section{Materials and methods}

DEG identification. The GSE21221 expression profile was downloaded from the gene expression omnibus (GEO) database. 
A

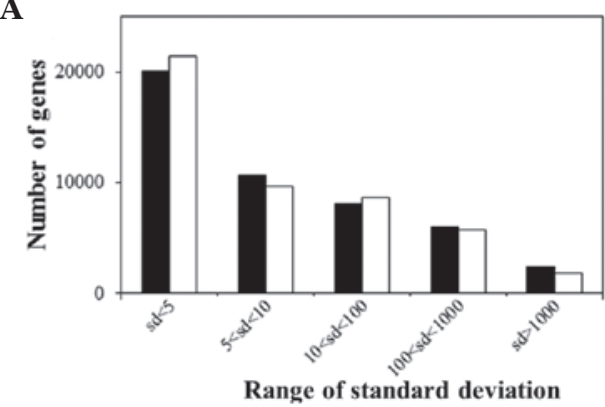

B

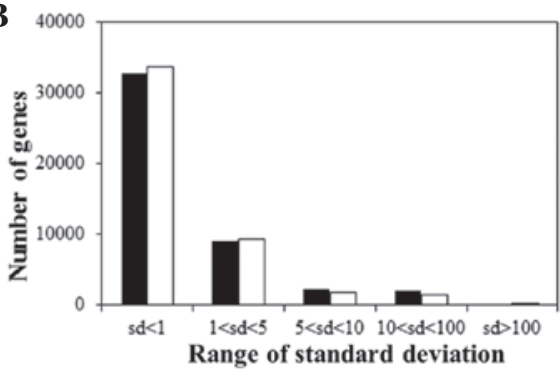

Figure 1. SD values of genes in (A) GPL2507 and (B) GPL10301. The x-axis represents the SD ranges. Black columns represent the SD values of genes in the healthy control group and the white column represent the SD values of genes in the Dupuytren's contracture group. SD, standard deviation.

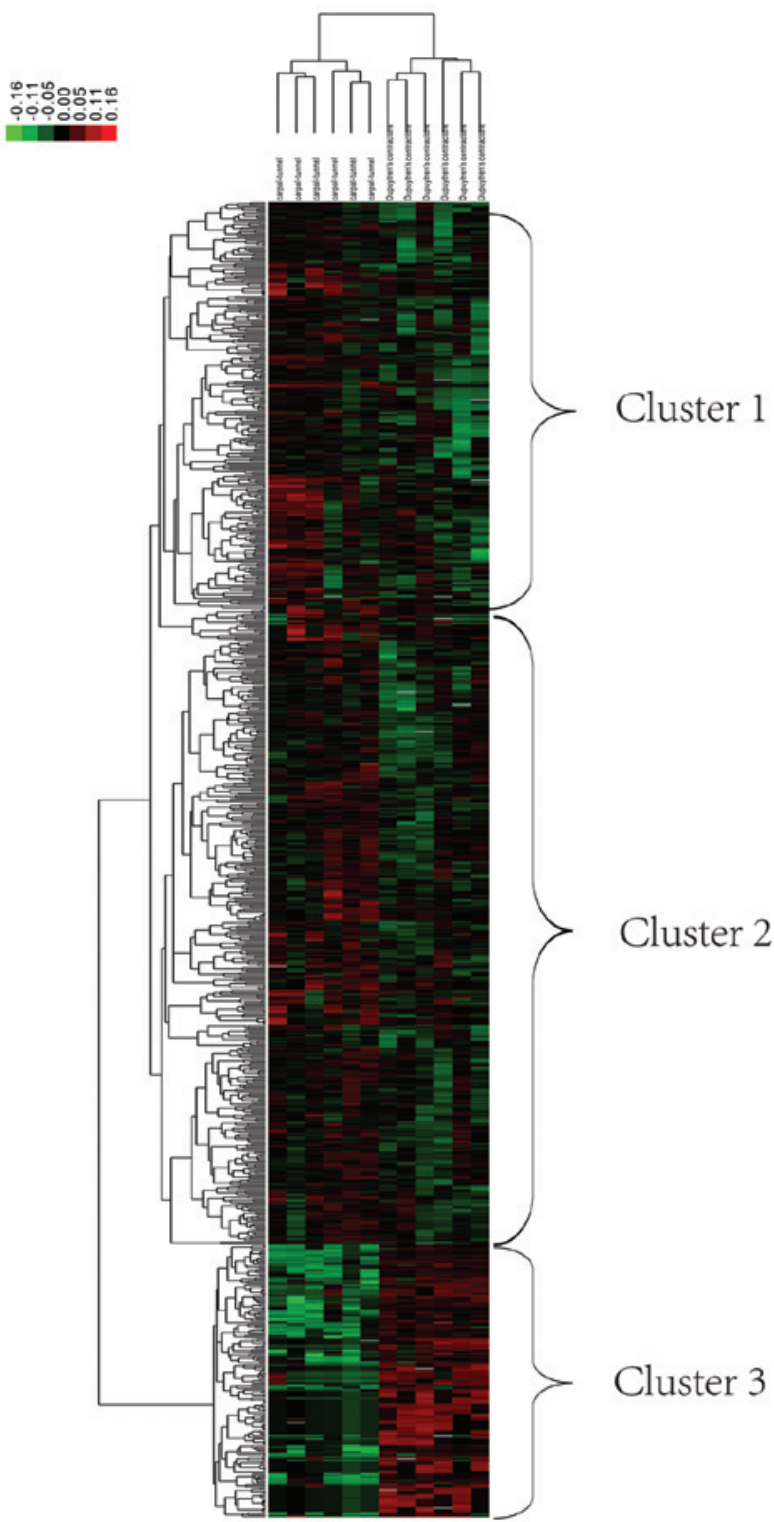

Figure 2 Heatmap of 775 genes. The $\mathrm{x}$-axis represents the genes and the $\mathrm{y}$-axis represents the samples. Green nodes represent downregulated genes and red nodes represent upregulated genes.

This included six samples from DC-derived fibroblasts and six control samples from carpal-tunnel-derived fibroblasts, and was conducted using GPL2507 Sentrix ${ }^{\circledR}$ Human-6 Expression
BeadChip and GPL10301 GE Healthcare CodeLink Human Whole Genome Bioarray (9). DEGs $(\mathrm{P}<0.05)$ were identified using the Distributed Intrusion Detection System (DIDS) (13) with R package version 1.1.

Hierarchical cluster analysis. Hierarchical cluster analysis for DEGs was performed in order to investigate the patterns of DEG classification and co-expression using TreeView (14). Genes whose expression values were present in $>80 \%$ of samples had a standard deviation (SD) $>2$ were selected. Expression values were logarithmically standardized using TreeView. The correlations in the similarity matrix were subsequently calculated and a hierarchical cluster analysis was performed.

Identification of contrary genes. A Pearson correlation coefficient was calculated using the co-express software (www.bioinformatics.lu/CoExpress), which analyzes the association between mRNA and miRNA expression. miRNA targets were predicted using TargetScan (http://www.targetscan.org/) and starBase (15). Genes with high expression values $<520$ and SD value $<560$ were removed. Between-experiment-normalization was then performed. Genes with a Pearson correlation coefficient $>0.9$ were defined as positive. Genes that were negatively associated with at least one gene in the positive gene group (Pearson correlation coefficient, $<-0.9$ and correlation coefficient, $<-0.5$ with all the genes in the positive gene group) were defined as negative. Genes in the positive and negative groups exhibited opposite expression patterns. Therefore, in the present study these genes are termed contrary genes, which exhibited opposite expression patterns in the positive and negative groups, and likely exhibited opposite functions.

Function annotation analysis of DEGs. A genecard database (16) was used in order to conduct function analysis of DEGs annotated with GO terms. Cluster analysis was conducted using reduce and visualize gene ontology (REViGO) (17), in order to identify the GO terms (similarity threshold: 0.7). Semantic similarity was measured using the SimRel algorithm (18).

System network analysis. A function network of GO terms was produced using the REViGO database (17), and network visualization was performed using cytoscape version 2.8 (19). 
Table I. Significantly correlated genes and correlation coefficients.

\begin{tabular}{llcccc}
\hline Node ID1 & Node ID2 & $\begin{array}{c}\text { Correlation } \\
\text { coefficient }\end{array}$ & Node ID1 & Node ID2 & $\begin{array}{r}\text { Correlation } \\
\text { coefficient }\end{array}$ \\
\hline CHI3L1 & EIF2S3 & -0.9273 & EPHX1 & ATF4 & -0.90163 \\
SFRP1 & MASP1 & 0.91333 & FAM12A & AKR1C2 & 0.9186 \\
SFRP1 & FBLN1 & 0.9048 & SAA1 & ATF4 & -0.9023 \\
FBLN1 & MASP1 & 0.93677 & NFE2L2 & AKR1C2 & 0.94147 \\
FBLN1 & CLU & 0.90097 & LAMA4 & AKR1C2 & 0.94183 \\
COL15A1 & RAB9A & -0.90623 & ITM2B & AKR1C2 & 0.92597 \\
RND3 & AKR1C2 & 0.92937 & RNF19A & ATF4 & -0.904 \\
EIF2S3 & AKR1C2 & 0.92647 & IFI16 & AKR1C2 & 0.90557 \\
DDX3X & AKR1C2 & 0.9201 & NPC1 & AKR1C2 & 0.91037 \\
\hline
\end{tabular}

CHI3LI, chitinase-3-like protein 1; SFRP, secreted frizzled-related protein 1; FBLN, fibulin; COL15A1, collagen type XV $\alpha$ 1; RND3, rho family GTPase 3; EIF2S3, eukaryotic translation initiation factor 2 subunit 3; DDX3X, DEAD (Asp-Glu-Ala-Asp) box helicase 3, X-linked; MASP1, mannan-binding lectin serine protease 1; CLU, clusterin; RAB, ras-related protein; AKR1C2, aldo-keto reductase family 1, member C2; EPHX1, epoxide hydrolase 1; FAM12A, family with sequence similarity 12, member A; SAA1, serum amyloid A1; NFE2L2, nuclear factor (erythroid-derived 2)-like 2; LAMA, laminin; ITM2B, integral membrane protein 2B; RNF19A, ring finger protein 19A; IFI16, $\gamma$-interferon-inducible protein 16; NPC1, Niemann-Pick disease, type C1; ATF4, activating transcription factor 4.

Table II. Cluster analysis of GO functions.

\begin{tabular}{llccc}
\hline GO ID & \multicolumn{1}{c}{ GO term } & Frequency $(\%)$ & Plot size & Uniqueness \\
\hline Cluster one & & & & \\
GO: 0001568 & Blood vessel development & 1.36 & 3.025 & 0.814 \\
GO: 0030097 & Hemopoiesis & 1.46 & 3.053 & 0.796 \\
GO: 0030099 & Myeloid cell differentiation & 0.64 & 2.694 & 0.781 \\
Cluster two & & & & \\
GO: 0008283 & Cell proliferation & 4.08 & 3.501 & 0.874 \\
GO: 0060548 & Negative regulation of cell death & 1.81 & 3.148 & 0.674 \\
GO: 0030307 & Positive regulation of cell growth & 0.21 & 2.21 & 0.722 \\
GO: 0008284 & Positive regulation of cell proliferation & 1.65 & 3.107 & 0.712 \\
Cluster three & & & & \\
GO: 0001933 & Negative regulation of protein phosphorylation & 0.49 & 2.584 & 0.666 \\
GO: 0006486 & Protein glycosylation & 0.73 & 2.755 & 0.787 \\
GO: 0016567 & Protein ubiquitination & 1.31 & 3.007 & 0.819 \\
GO: 0006468 & Protein phosphorylation & 5.22 & 3.608 & 0.786 \\
\hline
\end{tabular}

Frequency represents the frequency of the GO terms in the Genecards database and plot size represents the size of GO terms in the integral distribution after correction. Uniqueness represents the average similarity of the GO terms. GO, gene ontology.

A network analysis plugin (20) was then used in order to analyze the network topology and to identify the significant GO terms in the network.

\section{Results}

DEG identification. The distribution of SD values was used in order to indicate the level of homogeneity in the data. The results suggested that the data exhibited low levels of homogeneity (Fig. 1). A DIDS analysis was performed in order to identify DEGs in the DC samples that had significantly higher expression than normal range, this resulted in the identification of 801 DEGs $(\mathrm{P}<0.05)$.

Hierarchical cluster analysis. Using the hierarchical clustering method, significant DEGs (775) were identified among the 801 DEGs. Fig. 2 represents a heatmap produced using the cluster software, TreeView. The results suggested that the control and DC samples were clustered in three groups: Clusters one, two and three. In cluster one, genes in the control 

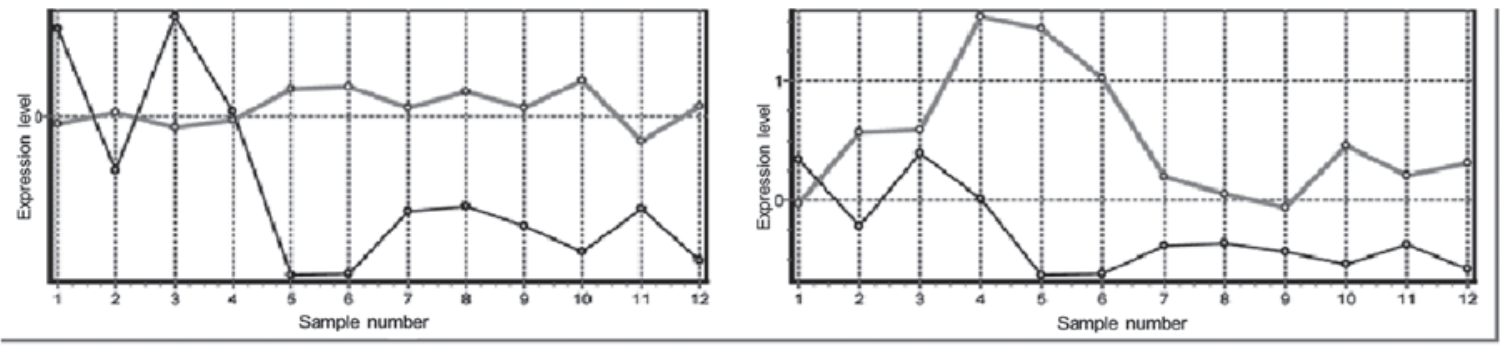

DDX3X-CHI3L1
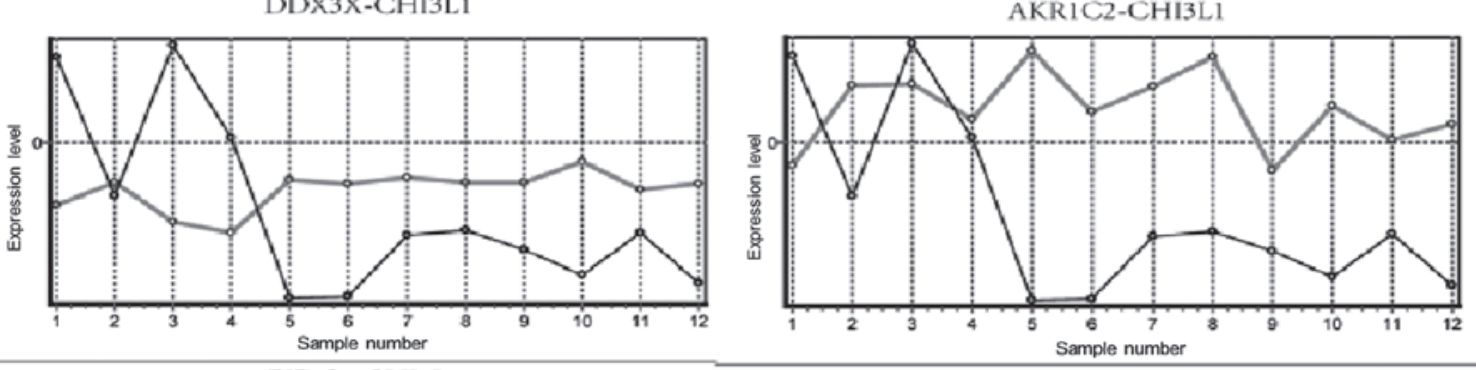

EIF2S3-CHI3L1
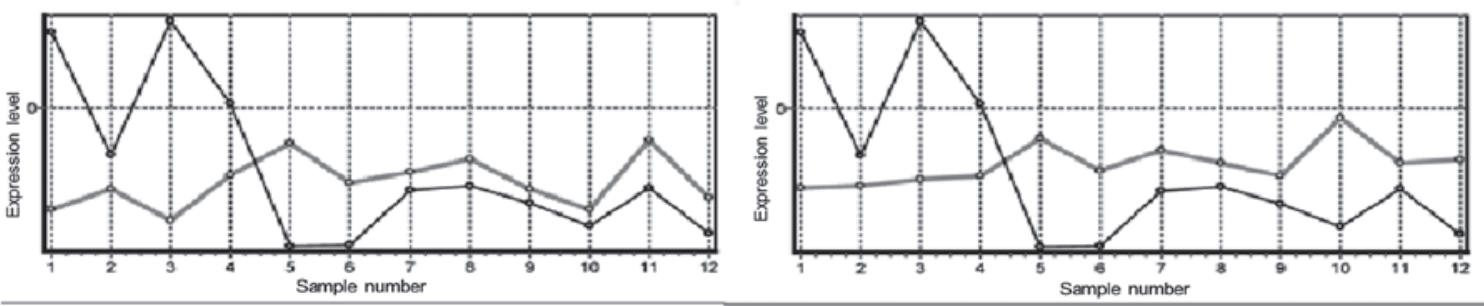

FAM129A-CHI3L1
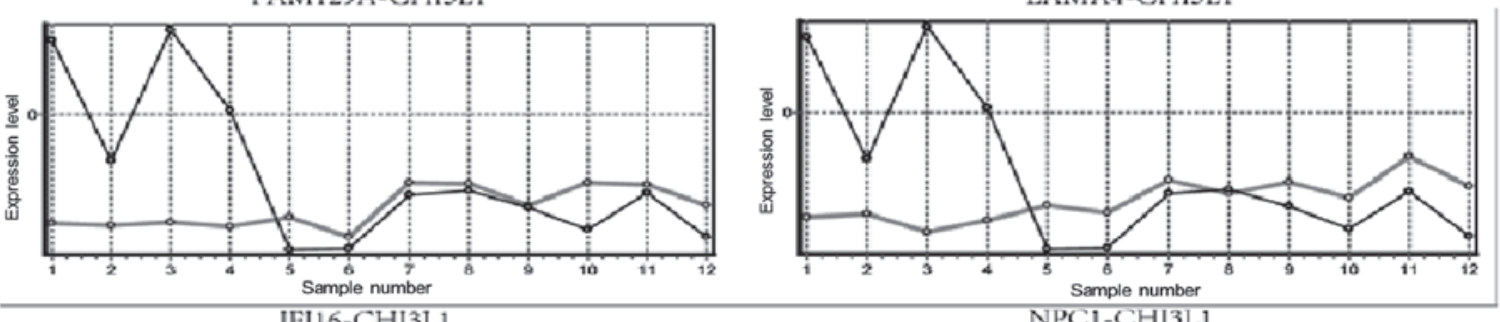

IFI16-CHI3L1

NPC1-CHI3L1
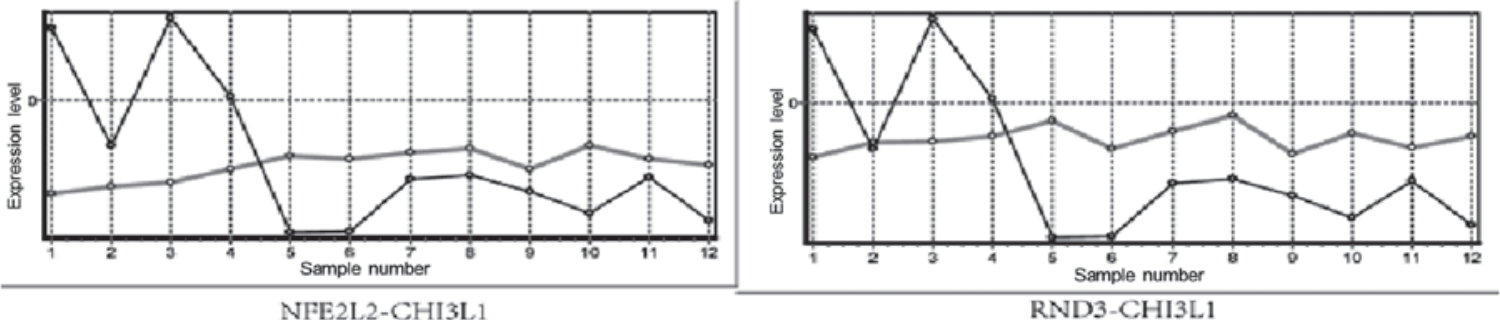

Figure 3. Expression levels of the ten genes from the positive group and the gene from the negative group, CHI3L1, in 12 samples (samples 1-6 are DC samples and samples 7-12 are healthy samples). The x-axis represents the 12 samples and the y-axis represents the expression levels following standardization. The black line represents the expression level of CHI3L1 and the grey line represents the expression levels of ten genes in the positive group. CHI3L1, chitinase-3-like protein 1; DC, Dupuytren's contracture; DDX3X, DEAD (Asp-Glu-Ala-Asp) box helicase 3 X-Linked; NFE2L2, nuclear factor erythroid 2-related factor 2; IFI16, $\gamma$-interferon-inducible protein 16; ITM2B, integral membrane protein 2B; NPC1, Niemann-Pick disease type C1; RND3, rho family GTPase 3; FAM129A, family with sequence similarity 129 A; EIF2S3, eukaryotic translation initiation factor 2 subunit $3 \gamma$; LAMA4, laminin $\alpha$; and AKR1C2, aldo-keto reductase family 1 member C2.

samples were upregulated, whereas those in the DC samples were significantly downregulated. In cluster two, genes in the control samples were upregulated and in the DC samples, upregulated and downregulated genes were observed. In cluster three, genes in the control samples were downregulated, whereas those in the DC samples were upregulated. In cluster three, DC and healthy samples were distinct from one another, whereas in clusters one and two, DC and healthy samples were not distinct. Therefore, genes associated with cluster three, may be useful for the development of diagnostic markers associated with DC.

Identification of contrary genes. Coexpress software was used in order to conduct a correlation analysis. The average expression value of the 801 DEGs was 523 and the average SD value was $2.24 \times 10^{2}$. Following filtering and standardization of the data, 98 contrary genes were identified. The average expression value of the 98 contrary genes was $3.45 \times 10^{3}$ and 


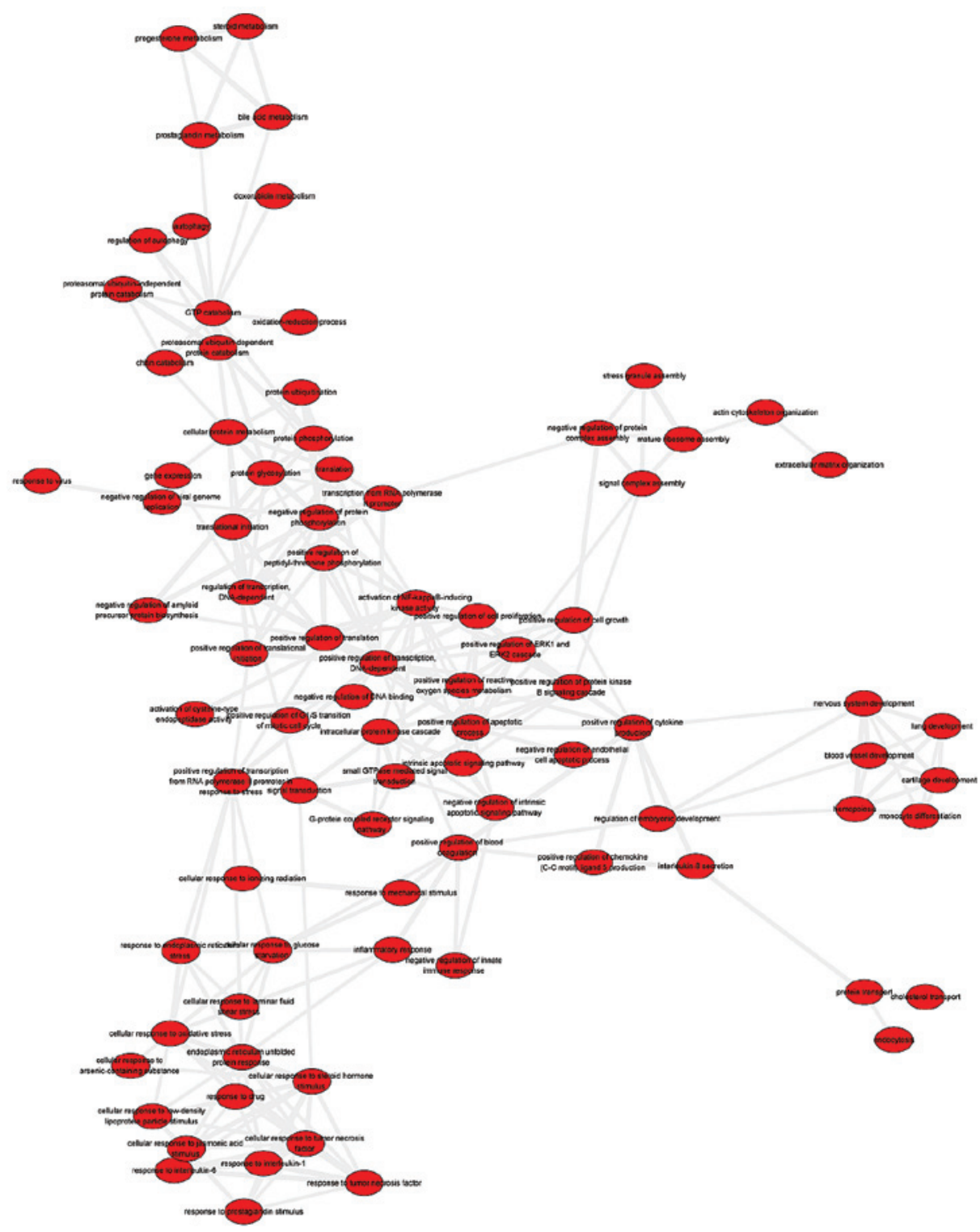

Figure 4. System network of $132 \mathrm{GO}$ terms. The nodes represent the GO terms and the edges represent the associations. GO, gene ontology.

Table III. GO terms exhibiting the ten highest degrees in the network which was constructed based on the GO function of CHI3L1 and ten positive genes.

\section{GO term}

Degree

Positive regulation of transcription 19

Activation of NF- $\kappa \mathrm{B}$-inducing kinase activity

Negative regulation of protein phosphorylation

16

Endoplasmic reticulum unfolded protein response

14

Cellular response to oxidative stress

14

12

Positive regulation of apoptotic process

12

Cellular response to steroid hormone stimulus

GTP catabolism

12

12

Positive regulation of translation

12

Positive regulation of ERK1 and ERK2 cascade

11

GO, gene ontology; CHI3L1, chitinase-3-like protein 1; ERK, extracellular signal-regulated kinase; GTP, guanosine-5'-triphosphate. the SD value was $5.56 \times 10^{2}$. Subsequently, 18 significantly correlated contrary gene pairs were identified (Table I). Chitinase-3-like protein 1 (CHI3L1) was negatively correlated with the following ten DEGs in the positive group: DEAD (Asp-Glu-Ala-Asp) box helicase 3; X-Linked (DDX3X); nuclear factor erythroid 2-related factor 2 (NFE2L2); $\gamma$-interferon-inducible protein 16 (IFI16); integral membrane protein 2B (ITM2B); Niemann-Pick disease, type C1 (NPC1); rho family GTPase 3 (RND3), family with sequence similarity 129 A (FAM129A); eukaryotic translation initiation factor 2, subunit $3 \gamma$ (EIF2S3), laminin, $\alpha 4$ (LAMA4); and aldo-keto reductase family 1 , member C2 (AKR1C2; Fig. 3).

Function annotation analysis. A GO function annotation analysis was performed for the CHI3L1 gene and the ten genes with which it was negatively correlated, which resulted in the identification of $132 \mathrm{GO}$ terms. The $132 \mathrm{GO}$ terms were subsequently grouped into three clusters corresponding to the clusters in Fig 2 (Table II). The results suggested that the genes in cluster one are involved in blood vessel development, 
hemopoiesis and myeloid cell differentiation. Genes in cluster two are involved in cell proliferation, and the negative and positive regulation of cell death. Genes in cluster three are involved in the negative regulation in protein phosphorylation, glycosylation, ubiquitination and phosphorylation.

System network analysis. A system network of the 132 GO terms was constructed (Fig. 4). GO terms exhibiting semantic similarities were removed. This resulted in $86 \mathrm{GO}$ terms and 257 edges, which represent the associations between GO terms. The GO terms, level of positive regulation of transcription and activation of nuclear factor- $\kappa \mathrm{B}(\mathrm{NF}-\kappa \mathrm{B})$-inducing kinase activity, exhibited degree levels of 19 and 16, respectively.

\section{Discussion}

In the present study, a DIDS analysis was conducted, which resulted in the identification of 801 DEGs. A positive and a negative group of DEGs were obtained. Opposing DEGs in the two groups are termed contrary genes in the present study. A network of GO terms was constructed, following a functional analysis, for the CHI3L1 gene and the ten genes with which it was negatively correlated (Table III).

Hierarchical clustering analysis was performed for 775 DEGs, which resulted in three clusters. Differences were observed between the expression patterns of genes in these three clusters. Contrary genes were identified based the correlation of gene expression level between the DEGs. CHI3L1 expression was found to be negatively correlated with the expression of ten DEGs in the positive group. CHI3L1 is a secreted glycoprotein, which may be termed YKL-40. Forrester et al (21) performed a genome-wide analysis of exon arrays, which demonstrated that CHI3L1 expression was higher in primary fibroblasts obtained from patients with Dupuytren's disease, compared with that in fibroblasts from healthy patients. In the present study, CHI3L1 expression was higher in the DC group than in the control group. Activation of $\mathrm{NF}-\kappa \mathrm{B}$-inducing kinase activity exhibited a high value in the GO term network. CHI3L1 has been shown to be induced by inflammatory cytokines, tumor necrosis factor- $\alpha$ (TNF- $\alpha$ ) and interleukin-1, in articular chondrocytes, and this induction requires sustained activation of $\mathrm{NF}-\kappa \mathrm{B}$ (22). Furthermore, it has been reported that the expression of TNF- $\alpha$ and interleukin-1 was significantly higher in patients with DC compared with that in controls (23). Therefore, CHI3L1 may be involved in the pathology of $\mathrm{DC}$, via the induction of NF- $\kappa \mathrm{B}$ kinase activity. However, the involvement of $\mathrm{CHI} 3 \mathrm{L1}$ in the development of DC requires further analysis. In the present study, the following ten genes were negatively correlated with CHI3L1: DDX3X, NFE2L2, IFI16, ITM2B, NPC1, RND3, FAM129A, EIF2S3, LAMA4 and AKR1C2. To the best of our knowledge, the present study is the first to demonstrate a potential association between these ten genes and DC development. DD3X (24), NFE2L2 (25), IFI16 (26), NPC1 (27), RND3 (28) and AKR1C2 (29) have been found to be positively correlated with inflammation. Therefore, these genes may be associated with DC development. However, the association between these genes and CHI3L1, and their involvement in DC, require further investigation, in order to understand the molecular mechanisms underlying DC development.
Positive regulation of transcription demonstrated the highest degree in the GO term network. The transcription factor gene, Zf9 is associated with DC pathogenesis (30). The expression of the contractile phenotype in Dupuytren's nodular cells is determined by the post-transcriptional regulation of $\alpha$-smooth muscle actin (31). Therefore, the regulation of transcription may be involved in the pathogenesis of DC.

In the present study, the expression of genes involved in DC was analyzed, and the term, contrary genes, was proposed. The expression of CHI3L1, was shown to be negatively correlated with that of ten other genes. These genes are involved in the positive regulation of transcription and in the activation of $\mathrm{NF}-\kappa \mathrm{B}$-inducing kinase activity. Therefore, the results of the present study may be of use in the development of diagnostic markers and treatment for DC.

\section{References}

1. Murphey MD, Ruble CM, Tyszko SM, Zbojniewicz AM, Potter BK and Miettinen M: From the archines of the AFIP: musculoskeletal fibromatoses: radiologic-pathologic correlation. Radiographics 29: 2143-2173, 2009.

2. Van Rijssen A and Werker P: Percutaneous needle fasciotomy in dupuytren's disease. J Hand Surg Br 31: 498-501, 2006.

3. Reilly RM, Stern PJ and Goldfarb CA: A retrospective review of the management of Dupuytren's nodules. J Hand Surg Am 30: 1014-1018, 2005

4. Loos B, Puschkin V and Horch RE: 50 years experience with Dupuytren's contracture in the Erlangen University Hospital - a retrospective analysis of 2919 operated hands from 1956 to 2006. BMC Musculoskelet Disord 8: 60, 2007.

5. Hurst LC and Badalamente MA: Nonoperative treatment of Dupuytren's disease. Hand Clin 15: 97-107, 1999.

6. Aggarwal BB and Shishodia S: Molecular targets of dietary agents for prevention and therapy of cancer. Biochem Pharmacol 71: 1397-1421, 2006.

7. Shimokawa $\mathrm{H}$ and Takeshita A: Rho-kinase is an important therapeutic target in cardiovascular medicine. Arterioscler Thromb Vasc Biol 25: 1767-1775, 2005.

8. Cordova A, Tripoli M, Corradino B, Napoli P and Moschella F: Dupuytren's contracture: an update of biomolecular aspects and therapeutic perspectives. Journ Hand Surg Br 30: 557-562, 2005.

9. Satish L, LaFramboise WA, O'Gorman DB, et al: Identification of differentially expressed genes in fibroblasts derived from patients with Dupuytren's Contracture. BMC Med Genomics 1: $10,2008$.

10. Mosakhani N, Guled M, Lahti L, et al: Unique microRNA profile in Dupuytren's contracture supports deregulation of $\beta$-catenin pathway. Mod Pathol 23: 1544-1552, 2010.

11. Lee LC, Zhang AY, Chong AK, Pham H, Longaker MT and Chang J: Expression of a novel gene, MafB, in Dupuytren's disease. J Hand Surg Am 31: 211-218, 2006.

12. Johnston P, Larson D, Clark IM and Chojnowski AJ: Metalloproteinase gene expression correlates with clinical outcome in Dupuytren's disease. J Hand Surg Am 33: 1160-1167, 2008.

13. Snapp SR, Brentano J, Dias GV, et al: DIDS (distributed intrusion detection system)-motivation, architecture, and an early prototype. In: Proceedings of the 14th National Computer Security Conference (Washington, DC, USA). Epstein J and Picciotto J (eds.): citeseer.ist.psu.edu/snapp91dids.html, pp 167-176, 1991.

14. Page RD: Visualizing phylogenetic trees using TreeView. Curr Protoc Bioinformatics 6, Unit 6.2, 2002.

15. Yang JH, Li JH, Shao P, Zhou H, Chen YQ and Qu LH: starBase: a database for exploring microRNA-mRNA interaction maps from Argonaute CLIP-Seq and Degradome-Seq data. Nucleic Acids Res 39: D202-D209, 2011.

16. Rebhan M, Chalifa-Caspi V, Prilusky J and Lancet D: GeneCards: a novel functional genomics compendium with automated data mining and query reformulation support. Bioinformatics 14: 656-664, 1998.

17. Supek F, Bošnjak M, Škunca $N$ and Šmuc T: REVIGO summarizes and visualizes long lists of gene ontology terms. PloS One 6: e21800, 2011. 
18. Zhang L and Hermanns H: Deciding simulations on probabilistic automata.In: Automated Technology for Verification and Analysis (ATVA), 5th International Symposium. Namjoshi KS et al (eds). Springer-Verlag, Heidelberg, pp 207-222, 2007.

19. Smoot ME, Ono K, Ruscheinski J, Wang PL and Ideker T: Cytoscape 2.8: new features for data integration and network visualization. Bioinformatics 27: 431-432, 2011.

20. Saito R, Smoot ME, Ono K, et al: A travel guide to Cytoscape plugins. Nat Methods 9: 1069-1076, 2012.

21. Forrester HB, Temple-Smith P,Ham S, de Kretser D, Southwick G and Sprung CN: Genome-wide analysis using exon arrays demonstrates an important role for expression of extra-cellular matrix fibrotic control and tissue remodelling genes in Dupuytren's disease. PLoS One 8: e59056, 2013.

22. Recklies AD, Ling H, White $C$ and Bernier SM: Inflammatory cytokines induce production of CHI3L1 by articular chondrocytes. J Biol Chem 280: 41213-41221, 2005.

23. Baird KS, Crossan JF and Ralston SN: Abnormal growth factor and cytokine expression in Dupuytren's contracture. J Clin Pathol 46: 425-428, 1993.

24. Wang L, Lawrence MS, Wan Y, et al: SF3B1 and other novel cancer genes in chronic lymphocytic leukemia. N Engl J Med 365: 2497-2506, 2011.

25. Saw C, Wu Q and Kong A: Anti-cancer and potential chemopreventive actions of ginseng by activating Nrf2 (NFE2L2) anti-oxidative stress/anti-inflammatory pathways. Chin Med 5: $37,2010$.
26. Veeranki S, Duan X, Panchanathan R, Liu H and Choubey D: IFI16 protein mediates the anti-inflammatory actions of the type-I interferons through suppression of activation of caspase-1 by inflammasomes. PLoS One 6: e27040, 2011.

27. Liao G, Cheung S, Galeano J, Ji AX, Qin Q and Bi X: Allopregnanolone treatment delays cholesterol accumulation and reduces autophagic/lysosomal dysfunction and inflammation in Npc1-/- mouse brain. Brain Res 1270: 140-151, 2009.

28. Kurtz KM and Breslin JW: Rnd3 accelerates termination of thrombin-induced Rho activation in endothelial cells. FASEB J (Meeting Abstract Suppl): 762.14, 2009.

29. Wang HW, Lin CP, Chiu JH, et al: Reversal of inflammation-associated dihydrodiol dehydrogenases (AKR1C1 and AKR1C2) overexpression and drug resistance in nonsmall cell lung cancer cells by wogonin and chrysin. Int J Cancer 120: 2019-2027, 2007.

30. Bayat A, Watson JS, Stanley JK, Ferguson MW and Ollier WE: Genetic susceptibility to dupuytren disease: association of Zf9 transcription factor gene. Plast Reconstr Surg 111: 2133-2139, 2003.

31. Verjee LS, Midwood K, Davidson D, Eastwood M and Nanchahal J: Post-transcriptional regulation of alpha-smooth muscle actin determines the contractile phenotype of Dupuytren's nodular cells. J Cell Physiol 224: 681-690, 2010. 